\title{
FIELD CONVEXITY OF A SQUARE MATRIX
}

B. N. MOYLS AND M. D. MARCUS

1. Introduction. Let $C$ be a complex $n$-square matrix and $z$ a complex $n$-vector. Let $(u, v)$ be the unitary inner product and set $f(z)$ $=(C z, z)$. The range of values of $f$ for $\|z\|=(z, z)^{1 / 2}=1$ is the field of values [1] of $C$ and we denote it by $F(C)$. Set $A=\left(C+C^{*}\right) / 2$, $B=\left(C-C^{*}\right) / 2 i, \phi(z)=(A z, z)$, and $\psi(z)=(B z, z)$. Then $f(z)=\phi(z)$ $+i \psi(z)$ and since $A$ and $B$ are Hermitian, $\phi$ and $\psi$ are real-valued.

It is known that $F(C)$ is convex in the plane [1]. To indicate briefly the proof of this fact note that $F\left(U C U^{*}\right)=F(C)$ for $U$ a unitary matrix. Hence we may assume $\phi(z)=\sum_{l=1}^{n} \mu_{i}\left|z_{i}\right|^{2}$ where the $\mu_{i}$ are the real eigenvalues of $A$. It is easy to show that for $\left\|z_{0}\right\|=1$, $\phi^{-1}\left(\phi\left(z_{0}\right)\right)$ is connected on the unit sphere and thus that $\psi\left(\phi^{-1}\left(\phi\left(z_{0}\right)\right)\right)$ is connected in the plane. This implies that $f\left(\phi^{-1}\left(\phi\left(z_{0}\right)\right)\right)$ is an interval and since convexity is unaltered by rotation we may conclude that every line intersects $F(C)$ in an interval.

In case $C$ is normal there exists a unitary matrix diagonalizing $C$ and hence we may assume

$$
f(z)=\sum_{i=1}^{n} \lambda_{i}\left|z_{i}\right|^{2}
$$

Thus $F(C)$ is the convex polygon spanned by the eigenvalues of $C$. For any $n$-square matrix $C$ let $P(C)$ be the polygon spanned by the eigenvalues and let $Q^{n}$ be the set of complex $n$-square matrices such that $P(C)=F(C)$. Also let $N^{n}$ be the set of all complex $n$-square normal matrices. We show that for $n \leqq 4, Q^{n}=N^{n}$; but for $n \geqq 5$, $N^{n} \subset Q^{n}$ and $Q^{n} \neq N^{n}$. However, we state necessary and sufficient conditions that $P(C)=F(C)$ for $C$ triangular and $n$ arbitrary. By Schur's Lemma [2] any matrix is unitarily similar to a triangular matrix and hence these conditions may be applied when $C$ is arbitrary by first reducing to triangular form.

\section{Results.}

Theorem 1. If $C$ is triangular and $C \in Q^{n}$ then, for $p<q, c_{p q}=0$ if either $c_{p p}$ or $c_{q q}$ is on the boundary of $P(C)$.

Proof. $C$ triangular implies

$$
f(z)=\sum_{q=1}^{n} \lambda_{q}\left|z_{q}\right|^{2}+\sum_{1=p<q}^{n} c_{p q} \bar{z}_{p} z_{q}
$$

Presented to the Society, December 29, 1954; received by the editors November 22, 1954. 
where $\lambda_{q}=c_{q q}$ are the eigenvalues of $C$. Let $\lambda_{p_{\alpha}}, \lambda_{p_{\beta}}$ be successive vertices of $P(C)$ taken in a counter clockwise sense. To see if $F(C)$ can lie on both sides of the segment joining $\lambda_{p_{\alpha}}$ and $\lambda_{p_{\beta}}$ we investigate the relationship between $R_{\alpha}(z)=\operatorname{Re}\left(\exp \left(-i \theta_{\alpha}\right) f(z)\right)$ and $\lambda_{\alpha p \alpha}$, where $\lambda_{\alpha q}=\operatorname{Re}\left(\exp \left(-i \theta_{\alpha}\right) \lambda_{q}\right)$ and $\theta_{\alpha}=\arg \left(\lambda_{p_{\beta}}-\lambda_{p_{\alpha}}\right)-\pi / 2$. Set $\left|z_{p}\right|=s_{p}$, $\left|c_{p q}\right|=r_{p q}$ and $\phi(p, q, \alpha)=\arg c_{p q}-\arg z_{p}+\arg z_{q}-\theta_{\alpha}$. Then

$$
\begin{aligned}
R_{\alpha}(z) & =\sum_{q=1}^{n} \lambda_{\alpha q} s_{q}^{2}+\sum_{p<q} \cos \phi(p, q, \alpha) r_{p q} s_{p} s_{q} \\
& =\lambda_{\alpha p_{\alpha}}-\sum_{q=1}^{n}\left(\lambda_{\alpha p_{\alpha}}-\lambda_{\alpha q}\right) s_{q}^{2}+\sum_{p<q} \cos \phi(p, q, \alpha) r_{p q} s_{p} s_{q}
\end{aligned}
$$

since $\sum_{q=1}^{n} s_{q}^{2}=1$. If $r_{p_{0} q_{0}} \neq 0$, where $\lambda_{p_{0}}$ is any eigenvalue on the segment joining $\lambda_{p_{\alpha}}$ and $\lambda_{p_{\beta}}$, set $z_{q}=0$ for $q \neq q_{0}$ or $p_{0}$, arg $z_{p_{0}}=\arg c_{p_{0} q_{0}}$ $+\arg z_{q_{0}}-\theta_{\alpha}$, and

$$
s_{p_{0}}>\frac{\left(\lambda_{\alpha p_{\alpha}}-\lambda_{\alpha q_{0}}\right)}{r_{p_{0} q_{0}}} s_{q_{0}}
$$

For such a vector $z$

$$
R_{\alpha}(z)>\lambda_{\alpha p_{\alpha}}=\lambda_{\alpha p_{0}}
$$

and we conclude that $C \notin Q^{n}$. Similarly $R_{\alpha}(z)>\lambda_{\alpha p_{\alpha}}$ for any $c_{q p_{0}} \neq 0$.

Corollary. $Q^{n}=N^{n}$ for $n \leqq 4$.

Proof. $N^{n} \subseteq Q^{n}$ follows from (1.1). Assume $A \in Q^{n}$ and transform $A$ to triangular form $C$ by a unitary matrix. For $n \leqq 4$ at most one eigenvalue of $A$ lies in the interior of $P(A)=P(C)$ and, by Theorem 1, $C$ is diagonal. Hence $A$ is normal and $A \in N^{n}$.

Similarly we have the following

Corollary. If at most one eigenvalue of $A$ lies in the interior of $P(A)$ then $A \in Q^{n}$ implies $A \in N^{n}$.

Denote by $\sum_{p}^{\prime}$ a sum obtained by deleting those indices $p$ for which $c_{p p}$ is on the boundary of $P(C)$. Similarly $\sum_{p<q}^{\prime}$ is the sum obtained by deleting those $(p, q)$ for which at least one of $p$ or $q$ is such that $c_{p p}$ or $c_{q q}$ is on the boundary of $P(C)$. Set

$$
\begin{aligned}
d_{\alpha q} & =\lambda_{\alpha p_{\alpha}}-\lambda_{\alpha q}, \\
z_{q} & =x_{q}+i y_{q}, \\
c_{p q} & =t_{p q}+i u_{p q} .
\end{aligned}
$$

Also define $S_{\alpha}(z)$ by the formula 


$$
\begin{aligned}
S_{\alpha}(z)= & \lambda_{\alpha p_{\alpha}}-\sum_{q}^{\prime} d_{\alpha q}\left(x_{q}^{2}+y_{q}^{2}\right) \\
& +\sum_{p<q}^{\prime}\left(t_{p q} \cos \theta_{\alpha}+u_{p q} \sin \theta_{\alpha}\right)\left(x_{p} x_{q}+y_{p} y_{q}\right) \\
& +\sum_{p<q}^{\prime}\left(t_{p q} \sin \theta_{\alpha}-u_{p q} \cos \theta_{\alpha}\right)\left(x_{p} y_{q}-y_{p} x_{q}\right) .
\end{aligned}
$$

THEOREM 2. If $C$ is triangular with $k$ eigenvalues lying in the interior of $P(C)$, then $C \in Q^{n}$ if and only if:

(a) $c_{p q}=0$ for $p<q$, when either $c_{p p}$ or $c_{q q}$ is on the boundary of $P(C) ;$ and

(b) the $2 k$ quadratic form $\lambda_{\alpha p \alpha}-S_{\alpha}(z)$ is positive semidefinite for each value of $\alpha$ corresponding to a side of $P(C)$.

Proof. From (2.2) and Theorem 1 we have

$$
R_{\alpha}(z) \geqq \lambda_{\alpha p_{\alpha}}-\sum_{q}^{\prime} d_{\alpha q} s_{q}^{2}+\sum_{p<q}^{\prime} \cos \phi(p, q, \alpha) r_{p q} s_{p} s_{q},
$$

and equality holds for a suitable choice of $z$. The result follows by substituting the relations (2.3) in (2.5), expanding, and noting (2.4).

Condition (b) of Theorem 2 may be expressed in terms of the positive semidefiniteness of the symmetric matrices $B_{\alpha}$ associated with $\lambda_{\alpha p_{\alpha}}-S_{\alpha}(z)$. The application of the criterion thus amounts to an inspection of the variations in sign of the coefficients of the characistic polynomials of the $B_{\alpha}$.

In some cases it is possible to choose the vector $z$ so that each $\cos \phi(p, q, \alpha)$ appearing in (2.5) is unity; then $\lambda_{\alpha p_{\alpha}}-S_{\alpha}(z)$ is a $k$-quadratic form in the $s_{q}$.

\section{REFERENCES}

1. W. V. Parker, Sets of complex numbers associated with a matrix, Duke Math• J. vol. 15 (1948) pp. 711-715.

2. I. Schur, Über die charakteristischen Würzeln einer linearen Substitution mit einer Anwendung auf die Theorie der Integralgleichungen, Math. Ann. vol. 66 (1909) pp. 488-510.

University of British Columbia 